\title{
Higgs results from ATLAS
}

\author{
Xin Chen ${ }^{1,2, a}$, On behalf of the ATLAS Collaboration \\ ${ }^{1}$ Department of Physics, Tsinghua University, Beijing 100084, China \\ ${ }^{2}$ Collaborative Innovation Center of Quantum Matter, Beijing, China
}

\begin{abstract}
The updated Higgs measurements in various search channels with ATLAS Run 1 data are reviewed. Both the Standard Model (SM) Higgs results, such as $H \rightarrow$ $\gamma \gamma, Z Z, W W, \tau \tau, \mu \mu, b \bar{b}$, and Beyond Standard Model (BSM) results, such as the charged Higgs, Higgs invisible decay and tensor couplings, are summarized. Prospects for future Higgs searches are briefly discussed.
\end{abstract}

\section{Introduction}

The discovery of the Higgs boson [1,2] by the ATLAS [3] and CMS experiments at the Large Hadron Collider (LHC) marks a major corner stone of the particle physics. Many new results have emerged after that, which span the aspects of its coupling strength and structure, spin/CP, mass, decay width and a broad range of BSM signatures. The most recent (up to the time of this publication) Higgs results using ATLAS 7 and $8 \mathrm{TeV} p$ - $p$ collision Run 1 data (about $25 \mathrm{fb}^{-1}$ in total) are reviewed here for different Higgs decay modes. An overview of future search prospects is given at the end of the paper.

\section{SM Higgs measurements}

The main Higgs production modes are gluon-gluon fusion $(g g H)$, vector-boson fusion (VBF), $V H$ associated and $t \bar{t} H$, with their cross sections in descending order. The productions diagrams are shown in Fig. 1. However, the search is most optimized according to different Higgs decays, since their SM backgrounds are vastly different. Productions, such as VBF, have additional background rejection features (e.g., forward jets). All production and decay modes have been searched for and combined to get the most sensitive results.

\section{$2.1 H \rightarrow \gamma \gamma$}

The branching ratio of $H \rightarrow \gamma \gamma$ [4] is only $0.23 \%$ at $m_{H}=125 \mathrm{GeV}$, but it has the advantage of a small background, which mainly consists of irreducible $\gamma \gamma$ (about 77\%), $\gamma+$ jet and dijet events. The first process has a large theoretical uncertainty. The latter two are mainly due to $\pi^{0}$ faking photons, whose rate is hard to model with simulation. These backgrounds are estimated from data using mass sidebands. This channel is optimized according to different production modes. The final signal strength

\footnotetext{
a e-mail: xin.chen@ cern.ch
} 


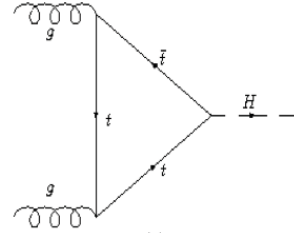

(1)

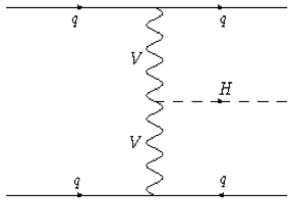

(2)

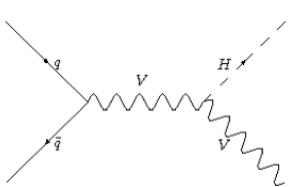

(3)

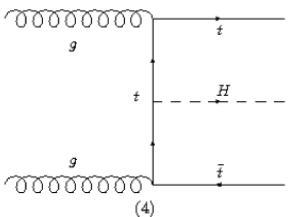

Figure 1. The Higgs production diagrams at LHC: (1) $g g H$, (2) VBF, (3) $V H$, (4) $t \bar{t} H$.

(measured rate divided by the SM prediction) based on Run 1 data is $\mu=1.17 \pm 0.27$. Fig. 2 shows the background composition and the $\gamma \gamma$ mass spectrum.
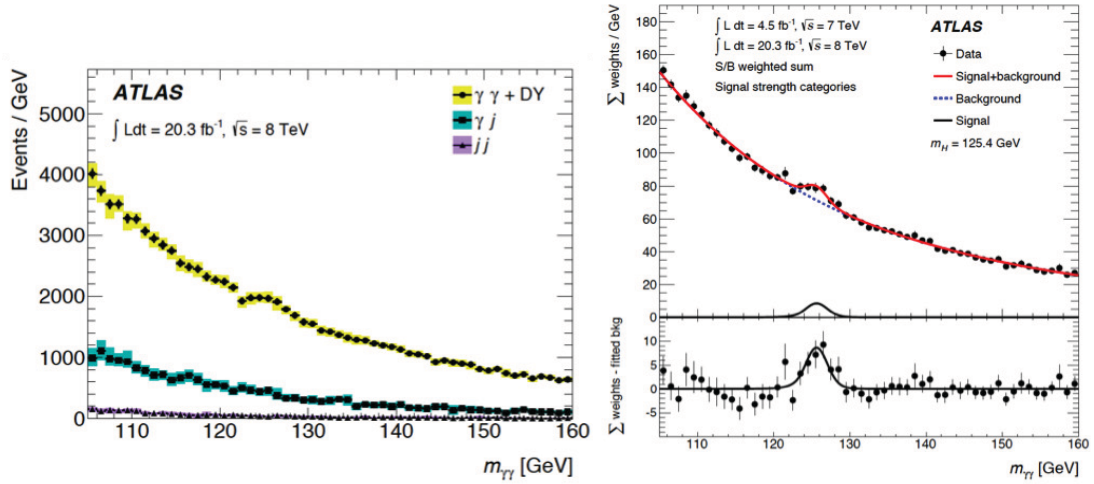

Figure 2. The background composition (left) and the $\gamma \gamma$ mass spectrum (right) in the $H \rightarrow \gamma \gamma$ channel. The mass spectrum is $S / B$-weighted from each category. Taken from Ref. [4].

\section{$2.2 H \rightarrow Z Z^{\star} \rightarrow 4 l$}

Although the branching ratio of $H \rightarrow Z Z^{\star} \rightarrow 4 l$ [5] is as small as $0.0125 \%$ at $m_{H}=125 \mathrm{GeV}$, it also has the smallest background, which mainly consists of $Z Z^{\star} \rightarrow 4 l$ and $Z b \bar{b}$. The latter can be estimated by inverting the lepton isolation or impact parameter cuts. To maximize acceptance, loose lepton identification is applied, and the lepton $p_{\mathrm{T}}$ is as low as $6 \mathrm{GeV}$. This channel has a very rich phase space (4-body decay), and all final state particles can be reconstructed. Thus, the Matrix Element (ME) method and Boosted Decision Trees (BDT) can be used to extract the signal strength and other properties. Fig. 3 shows the 2-D $m_{4 l}$ versus BDT and the $m_{4 l}$ distributions. This channel is optimized for different production modes as well. The signal strength measured is $1.44_{-0.31}^{+0.34}(\mathrm{stat})_{-0.11}^{+0.21}$ (sys).

\subsection{Higgs mass measurements}

The Higgs mass measurements are only feasible in the $H \rightarrow \gamma \gamma$ and $H \rightarrow Z Z^{\star} \rightarrow 4 l$ channels [6]. The results put a strong constraint on BSM models such as the Super Symmetry (SUSY). The $H \rightarrow \gamma \gamma$ mass is determined by the photon calibration and calo-pointing (calorimeter cell orientation and the 

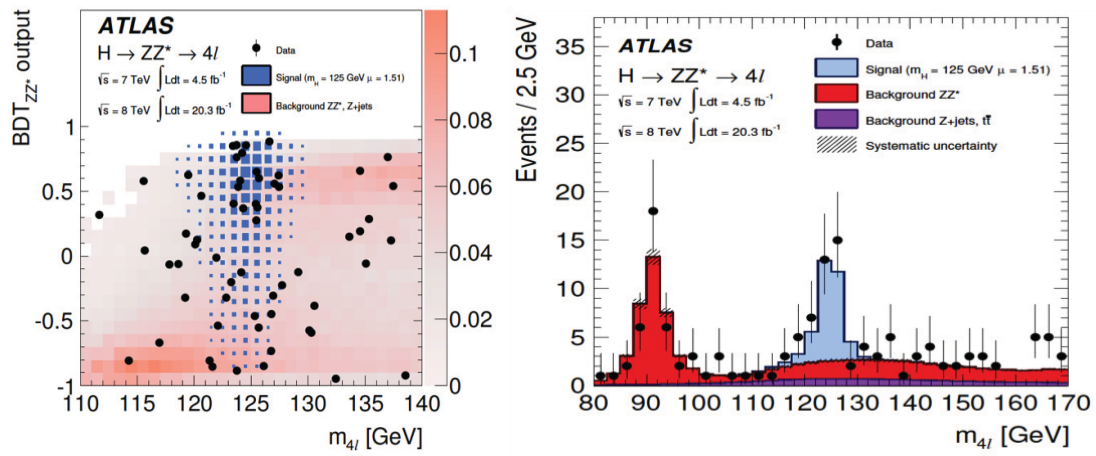

Figure 3. The $m_{4 l}$ versus BDT 2-D distribution (left) and the $m_{4 l}$ distribution (right) in the $H \rightarrow Z Z^{\star} \rightarrow 4 l$ channel. Taken from Ref. [5].

primary vertex are used to measure the photon direction). The events are categorized into different photon categories (converted or unconverted, central or in the transition region) to get the best mass resolution. The final result is $125.98 \pm 0.42$ (stat) \pm 0.28 (sys). The $H \rightarrow Z Z^{\star} \rightarrow 4 l$ mass is obtained by 2 -D fit to mass and BDT distributions with a $Z$ mass constraint. The final result is $124.51 \pm 0.52$ (stat) \pm 0.06 (sys). The combined mass of the two channels is $125.36 \pm 0.37$ (stat) \pm 0.18 (sys). It is worth to note that the two mass measurements are still compatible within $2 \sigma$ level.

\section{$2.4 H \rightarrow W W^{\star} \rightarrow 2 l 2 v$}

The branching ratio of $H \rightarrow W W^{\star} \rightarrow 2 l 2 v(l=e, \mu)$ [7] is at $1 \%$ level at $m_{H}=125 \mathrm{GeV}$. However, the background is also more severe, which is dominated by top (t$t \bar{t}$ and single top), Drell-Yan (for same-flavor leptons) and $W W$. To maximize the sensitivity, the analysis is divided into 0,1 and 2-jet categories, as the background composition is different in different jet bins (see the $N_{\text {jet }}$ distribution in Fig. 4). Thanks to the spin correlation between the two $W$ bosons, the two leptons from their decay tend to be aligned with each other. This feature leads to large $p_{\mathrm{T}}^{l l}$, small $m_{l l}$ and $\Delta \Phi_{l l}$, which can help suppressing the Drell-Yan background. It is not possible to construct the Higgs mass, but a transverse mass, defined as $m_{\mathrm{T}}=\sqrt{\left(E_{\mathrm{T}}^{l l}+p_{\mathrm{T}}^{\nu v}\right)^{2}-\left|\mathbf{p}_{\mathrm{T}}^{l l}+\mathbf{p}_{\mathrm{T}}^{\nu v}\right|^{2}}$, can be used. The $m_{\mathrm{T}}$ distribution in the most sensitive $e \mu+0$-jet category (dominated by $g g H$ ) is shown in Fig. 4. The final signal strength is $1.09_{-0.21}^{+0.23}$ in this channel.

\section{$2.5 H \rightarrow \tau \tau$}

The branching ratio of $H \rightarrow \tau \tau$ [8] is $6.3 \%$ at $m_{H}=125 \mathrm{GeV}$, and it has a relatively clean background. Due to the smallness of the $\tau$ mass, the visible ( $e / \mu$ or pions) and invisible (neutrinos) decay products tend to be aligned. A likelihood based algorithm, named MMC [9], is used to construct the di- $\tau$ mass. The analysis is optimized according to the leptonic or hadronic $\tau$ decay modes: $\tau_{\text {lep }} \tau_{\text {lep }}, \tau_{\text {lep }} \tau_{\text {had }}, \tau_{\text {had }} \tau_{\text {had }}$. Due to the large uncertainty from the missing transverse momentum $\left(E_{\mathrm{T}}^{\mathrm{miss}}\right)$, the MMC mass resolution is about $20 \mathrm{GeV}$. As the $Z$ and $H$ mass peaks are close under this resolution, it is very important to model the $Z \rightarrow \tau \tau$ background well. This is realized by taking the $Z \rightarrow \mu \mu$ data events, and replacing the muon by simulated $\tau$ decay products. A BDT-based analysis is used to extract the signal. Fig. 5 shows the MMC distribution with all sub-channels combined. 

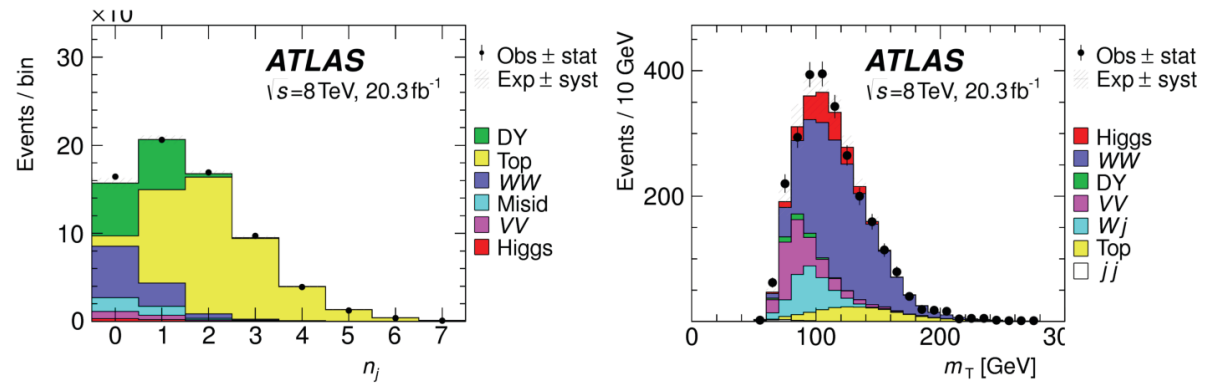

Figure 4. The jet multiplicity in the $e \mu$ category (left) and transverse mass in the $e \mu+0$-jet category (right) in the $H \rightarrow W W^{\star} \rightarrow 2 l 2 v$ channel. Taken from Ref. [7].

The enhancement corresponds to a signal strength of $1.43_{-0.26}^{+0.27}(\text { stat })_{-0.25}^{+0.32}$ (sys) \pm 0.09 (theo), or a $4.5 \sigma$ discovery.
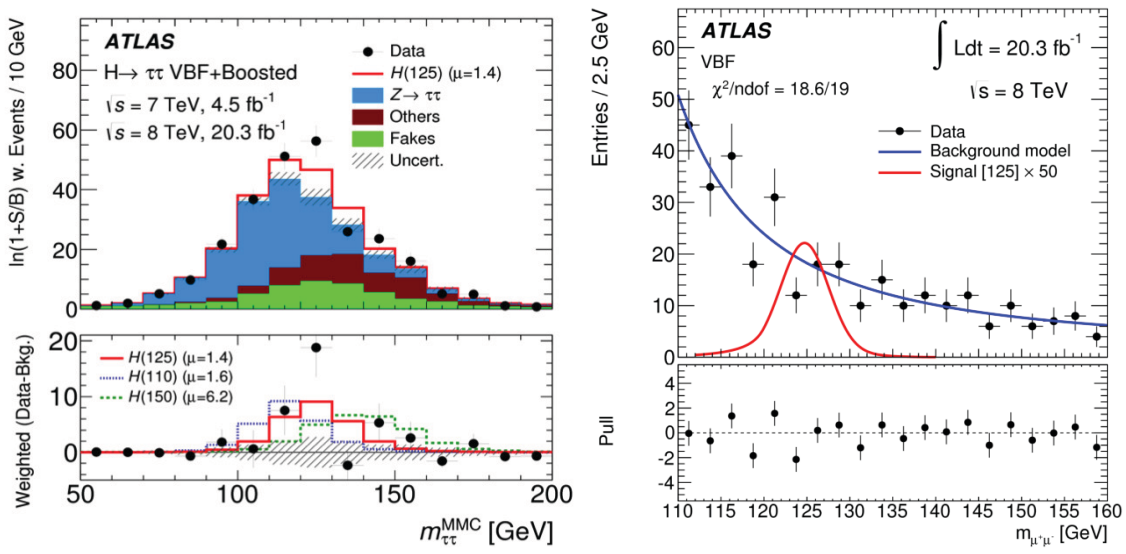

Figure 5. Left: the MMC di- $\tau$ mass weighted by weighted by $\ln (1+S / B)$ in the $H \rightarrow \tau \tau$ channel. Right: the $\mu \mu$ mass spectrum in the $H \rightarrow \mu \mu$ channel. Taken from Ref. [8, 10].

\section{$2.6 H \rightarrow \mu \mu$}

The branching ratio of $H \rightarrow \mu \mu$ [10] is very small, and overwhelmed by the Drell-Yan $Z \rightarrow \mu \mu$ background. The analysis focuses on the VBF production, and is divided into several $p_{\mathrm{T}}$ bins of Higgs. The current observed upper limit (at 95\% CL) of the signal strength is 7.0. The $\mu \mu$ mass spectrum is shown in Fig. 5.

\section{$2.7 H \rightarrow b \bar{b}$}

The $H \rightarrow b \bar{b}$ has the largest branching ratio $\left(57.7 \%\right.$ at $\left.m_{H}=125 \mathrm{GeV}\right)$, but suffers from a large QCD $b \bar{b}$ background. Together with the di- $\tau$ channel, they provide direct measurements of the Higgs 
Yukawa couplings. The main search channels are $H \rightarrow b \bar{b}$ production associated with a vector boson $(V H)$ [11] or a $t \bar{t}$ pair $(t \bar{t} H)$ [12]. Depending on the boson $(W / Z)$ decay modes, the former is further divided in to $0 / 1 / 2$-lepton and a few boson $p_{\mathrm{T}}$ categories, with signal optimization in each. The major backgrounds for $V+H \rightarrow b \bar{b}$ are $W / Z+b \bar{b} / c \bar{c}$, and BDT is used to extract the signal. The $b \bar{b}$ mass with backgrounds subtracted, except for $V+Z \rightarrow b \bar{b}$, is shown in Fig. 6 . The signal strength is $0.51_{-0.30}^{+0.31}$ (stat) ${ }_{-0.22}^{+0.25}$ (sys). In the $t \bar{t}+H \rightarrow b \bar{b}$ channel, the analysis is divided into 1- and 2-lepton modes, and several bins of different jet/b-jet numbers. Since the signal has $\geq 4(\geq 6)$ jets in the 1-lepton (2-lepton) mode, and $\geq 4 b$-jets, the high (low) jet/ $b$-jet multiplicity bins are used as signal (background control) regions. Neural Network (NN) is used to extract the signal, and in the case of 1-lepton mode, ME method is also used for the NN input. The NN distribution in one of the most sensitive regions is shown in Fig. 6 . The signal strength is $1.5 \pm 1.1$. This channel provides a direct $t \bar{t} H$ coupling measurement that otherwise can only be obtained implicitly from $g g H$ production.
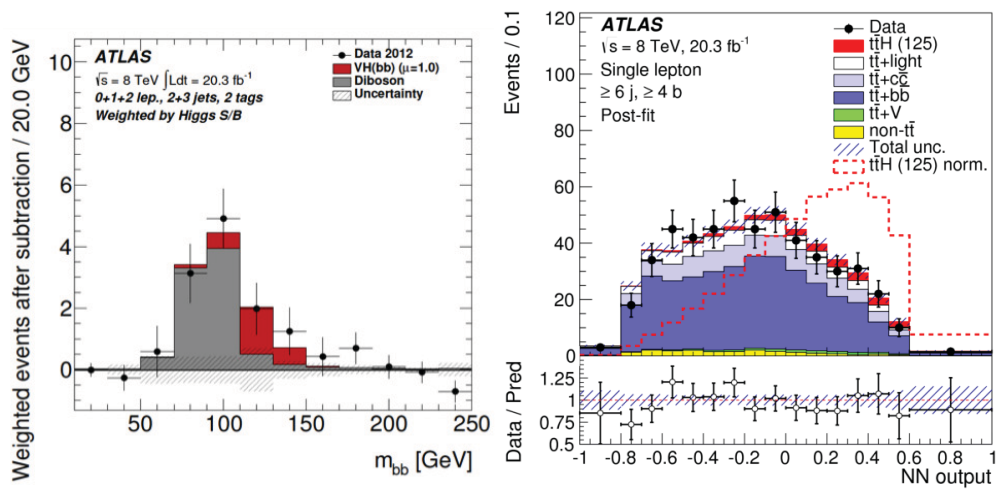

Figure 6. Left: the weighted $b \bar{b}$ mass in the $V+H \rightarrow b \bar{b}$ channel with all $V$ decay modes combined. All backgrounds are subtracted except for $V+Z \rightarrow b \bar{b}$. Right: the NN distribution after fit in the $t \bar{t}+H \rightarrow b \bar{b}$ channel and 1-lepton $+\geq 6$ jets $+\geq 4 b$-jets signal region. Taken from Ref. [11, 12].

\subsection{Higgs coupling combination}

The statistically combined signal strength for all decay channels is $1.18_{-0.14}^{+0.15}$ [13]. Fig. 7 shows the ME level coupling strength factors for different $H V V$ and $H f \bar{f}$ couplings. The double minima are due to relative signs of $\kappa_{W}$ and $\kappa_{t}$ in the $H \rightarrow \gamma \gamma$ loop. However, the global minimum is still compatible with the SM prediction $\left(\kappa_{V}=\kappa_{f}=1\right)$ within $1 \sigma$. Fig. 7 also shows the Higgs coupling as a function of different particle mass. The fact that all the measurements lie around a straight line is a confirmation of the SM Higgs mechanism.

\section{BSM Higgs searches}

The BSM Higgs searches are carried out in many aspects: direct searches of new Higgs scalars such as the charged Higgs, Higgs coupling to invisible particles and the altering of its decay width, new coupling structures of Higgs coupling to bosons and fermions. The highlights of a few search results are given below. 

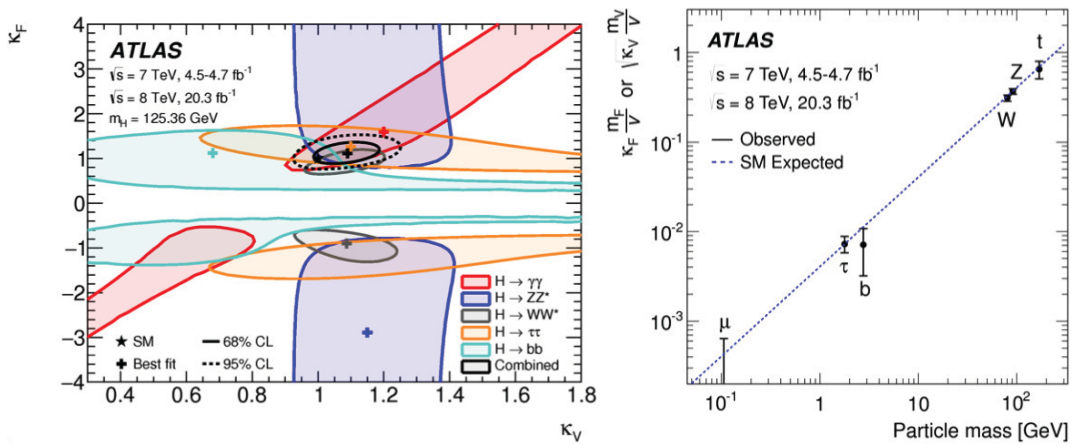

Figure 7. Left: the Higgs coupling strength for different particles with 1 and $2 \sigma$ contours. All bosons and fermions share a common coupling strength factor, $\kappa_{V}$ and $\kappa_{f}$, respectively, Right: the Higgs coupling as a function of different particle mass. Taken from Ref. [13].

\subsection{Charged Higgs search}

Charged Higgs is a benchmark of two Higgs doublet models. Its search is carried out in two phase space regions depending on whether its mass is below or above the top quark mass [14]. The main production diagrams in these two regions are shown in Fig. 8. The decay of $H^{ \pm}$searched for is $H^{ \pm} \rightarrow \tau^{ \pm} v$. In order to construct the transverse mass, all-hadronic decays of top and the hadronic $\tau$ decay are required. These events are recorded by $\tau+E_{\mathrm{T}}^{\text {miss }}$ triggers. At least 3 or 4 jets, one $b$-tagged jet and a large $E_{\mathrm{T}}^{\text {miss }}$ are required. The background is divided into real or fake $\tau_{\text {had }}$ events, with the former estimated from $W \rightarrow \mu \nu$ data events (replacing the muon by simulated $\tau$ decay). Fig. 9 shows the model independent upper limits on $\mathrm{B}\left(t \rightarrow b H^{ \pm}\right) \times \mathrm{B}\left(H^{ \pm} \rightarrow \tau^{ \pm} v\right)$ or $\sigma_{H^{ \pm}} \times \mathrm{B}\left(H^{ \pm} \rightarrow \tau^{ \pm} v\right)$, and the exclusion regions in a bench mark point in the MSSM parameter space, $m_{h}^{\bmod \pm}$, whose light Higgs mass is consistent with $125 \mathrm{GeV}$. The region with $H^{ \pm}$mass close to the top is not considered as it is phase space suppressed.
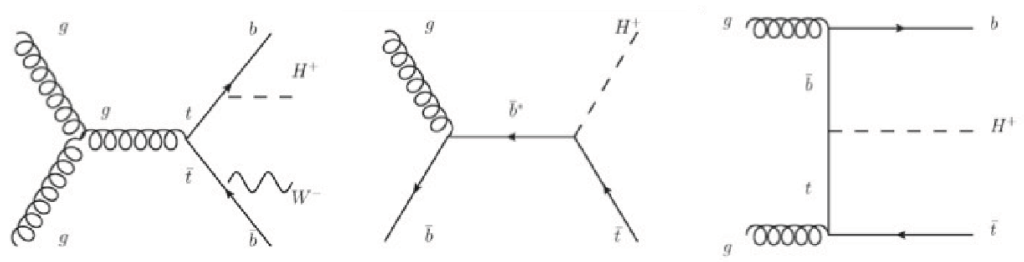

Figure 8. The production diagrams for charged Higgs in the low (left) and high (middle, right) mass regions. Taken from Ref. [14].

\subsection{Off-shell Higgs}

The on-shell production of diboson $(W W / Z Z)$ can be used to probe the off-shell Higgs [15]. Unfortunately, similar to the case of double Higgs production, it receives the interference from Higgs-less box diagram diboson productions, as shown in Fig. 10. Therefore, they are considered together as the 

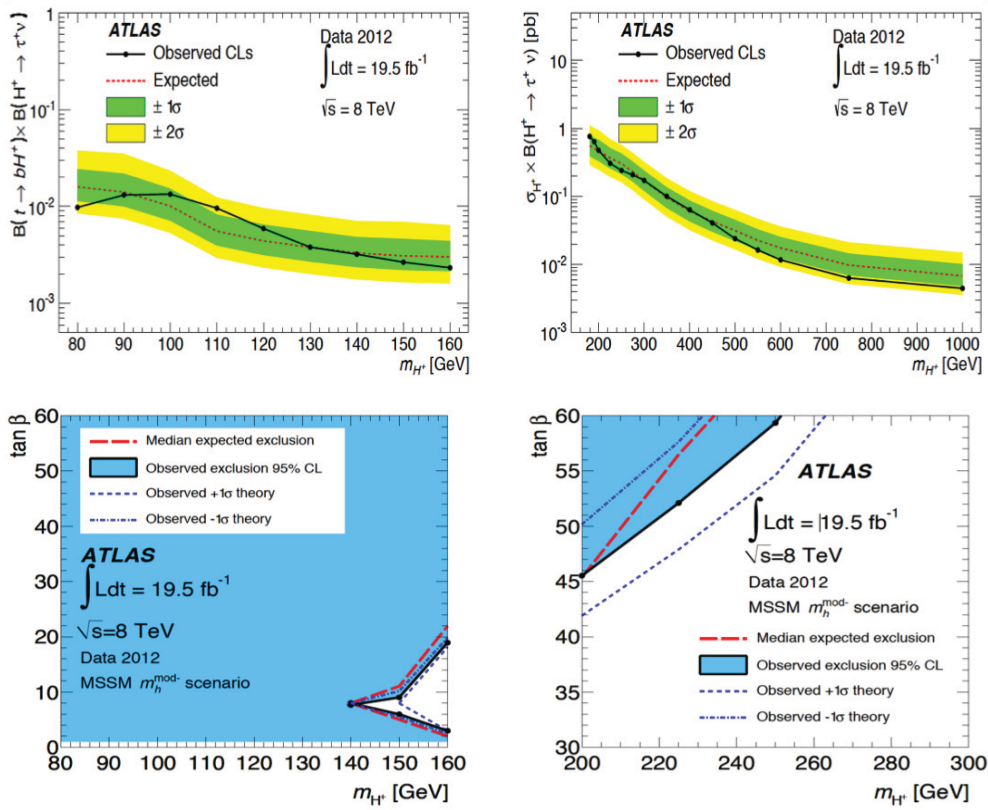

Figure 9. The 95\% $\mathrm{CL}$ upper limit on $\mathrm{B}\left(t \rightarrow b H^{ \pm}\right) \times \mathrm{B}\left(H^{ \pm} \rightarrow \tau^{ \pm} v\right)$ or $\sigma_{H^{ \pm}} \times \mathrm{B}\left(H^{ \pm} \rightarrow \tau^{ \pm} v\right)$ for the low (upper left) and high (upper right) mass regions. The $95 \% \mathrm{CL}$ exclusion regions in the $m_{H^{ \pm}}-\tan \beta$ plane in the MSSM $m_{h}^{\text {modt }}$ model in the low (bottom left) and high (bottom right) mass regions. Taken from Ref. [14].

signal. The off-shell Higgs signal strength is expressed as a function of center-of-mass scale $\hat{s}$ and the gluon and boson coupling strength factors $\left(\kappa_{g}\right.$ and $\left.\kappa_{V}\right)$ :

$$
\mu_{\text {off-shell }}(\hat{s})=\frac{\sigma_{\text {off-shell }}^{g g \rightarrow H^{\star} \rightarrow V V}(\hat{s})}{\sigma_{\text {off-shell,SM }}^{g g \rightarrow H^{\star} \rightarrow V V}(\hat{s})}=\kappa_{g, \text { off-shell }}^{2}(\hat{s}) \kappa_{V, \text { off-shell }}^{2}(\hat{s}) .
$$

To suppress the other diboson background, in the $Z Z \rightarrow 4 l$ decay, ME method with MCFM [16] is used, while in the $Z Z \rightarrow 2 l 2 v$ and $W W \rightarrow e v \mu v$ decays, the transverse mass is used as the discriminant. Unfortunately, because the cross section for box process $g g \rightarrow V V$ is not well known, the final results are expressed as a function of $R_{H^{\star}}^{B}=\kappa(g g \rightarrow V V) / \kappa\left(g g \rightarrow H^{\star} \rightarrow V V\right)$. The 95\% CL upper limit on $\mu_{\text {off-shell }}$ is shown in Fig. 11. For $R_{H^{\star}}^{B}=1$, the observed (expected) upper limit is 6.2 (8.1). Subject to a number of assumptions, when the off-shell results are combined with the on-shell signal strength measurement, which is defined as

$$
\mu_{\text {on-shell }}=\frac{\sigma_{\text {on-shell }}^{g g \rightarrow H}}{\sigma_{\text {on-shell,SM }}^{g g \rightarrow H V}}=\frac{\kappa_{g, \text { on-shell }}^{2} \kappa_{V, \text { on-shell }}^{2}}{\Gamma_{H} / \Gamma_{H}^{\mathrm{SM}}},
$$

a measurement of the Higgs width can be obtained. The fitted $-2 \ln L$ as a function of $\Gamma_{H} / \Gamma_{H}^{\mathrm{SM}}$ is also given in Fig. 11 at $R_{H^{\star}}^{B}=1$. The observed (expected) upper limit on $\Gamma_{H} / \Gamma_{H}^{\mathrm{SM}}$ is 5.5 (8.0). 


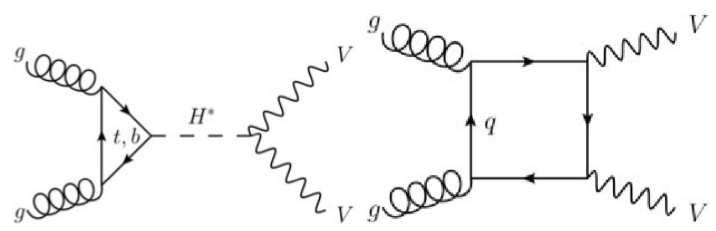

Figure 10. The on-shell production diagrams of diboson that interfere with each other. The left diagram corresponds to the off-shell Higgs production signal. Taken from Ref. [15].
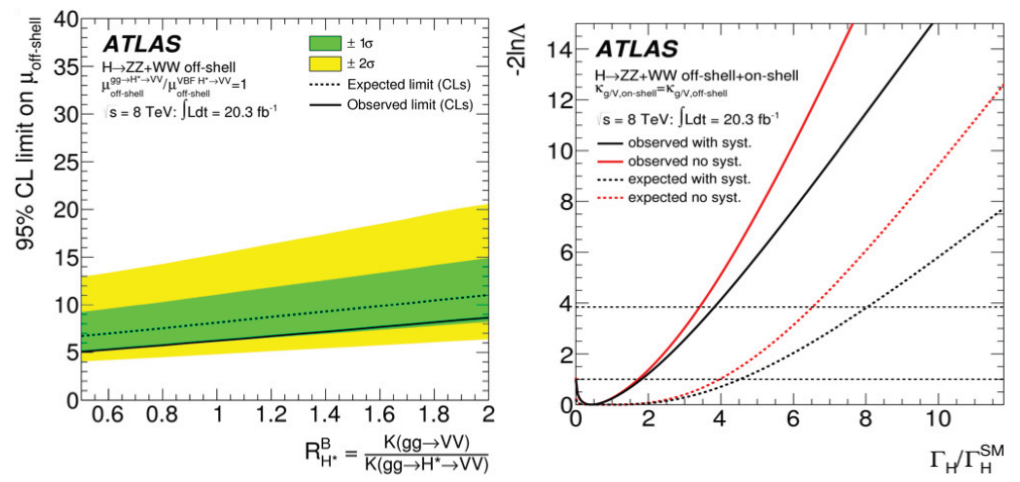

Figure 11. Left: the $95 \%$ CL limit on $\mu_{\text {off-shell }}$ as a function of $R_{H^{\star}}^{B}$. Right: the $-2 \ln L$ as a function of $\Gamma_{H} / \Gamma_{H}^{\mathrm{SM}}$. Taken from Ref. [15].

\section{3 $H \rightarrow$ invisible}

Models like SUSY predict dark matter particles that may couple to the Higgs boson. Direct searches for $H \rightarrow$ invisible decay are carried out in the $V H$ and VBF productions. In the $V H$ mode, the search for $\mathrm{ZH} \rightarrow l l+$ invisible yields $\mathrm{BR}(H \rightarrow$ invisible $)<0.75$ (0.62) [17] for the observed (expected) limit, and the search for $V H \rightarrow$ dijet+invisible gives $\mathrm{BR}(H \rightarrow$ invisible $)<0.78(0.86)$ [18] for the observed (expected). In the VBF search mode, signal events are recorded by the $E_{\mathrm{T}}^{\text {miss }}$ trigger, and the dominant background $(Z \rightarrow v v+$ jets and $W \rightarrow l v+$ jets) is estimated with dedicated $Z \rightarrow l l$ and $W \rightarrow l v$ control samples. The Run 1 data gives 539 events in the signal region, while 579 events are expected. An observed (expected) limit of $\mathrm{BR}(H \rightarrow$ invisible $)<0.29$ (0.35) [19] is obtained. When all three channels are combined, the observed (expected) limit is $\operatorname{BR}(H \rightarrow$ invisible $)<0.25$ (0.27) [20], for which the sensitivity is dominated by the VBF channel.

\subsection{Higgs tensor coupling}

Using Effective Field Theory (EFT), a general $H V V$ coupling can be expressed as (for spin- 0 ):

$$
\begin{aligned}
\mathcal{L}_{0}= & c_{\alpha} \kappa_{\mathrm{SM}}\left[\frac{1}{2} g_{H Z Z} Z_{\mu} Z^{\mu}+g_{H W W} W_{\mu}^{+} W^{-\mu}\right] X_{0}+ \\
& \left\{-\frac{1}{4} \frac{1}{\Lambda}\left[c_{\alpha} \kappa_{H Z Z} Z_{\mu \nu} Z^{\mu v}+s_{\alpha} \kappa_{A Z Z} \tilde{Z}^{\mu v}\right]-\frac{1}{2} \frac{1}{\Lambda}\left[c_{\alpha} \kappa_{H W W} W_{\mu \nu}^{+} W^{-\mu v}+s_{\alpha} \kappa_{A W W} W_{\mu \nu}^{+} \tilde{W}^{-\mu v}\right]\right\} X_{0},
\end{aligned}
$$

where the first line corresponds to the SM CP-even coupling, denoted $0^{+}$, the $\kappa_{H V V}\left(\kappa_{A V V}\right)$ terms in the second line represent the BSM CP-even (CP-odd) couplings, denoted $0_{h}^{+}\left(0^{-}\right)$, the $\tilde{V}=\frac{1}{2} \epsilon^{\mu \nu \rho \sigma} V_{\rho \sigma}$ 
is the dual tensor, $c_{\alpha}=\cos \alpha, s_{\alpha}=\sin \alpha, \alpha$ is the mixing angle between CP-even and CP-odd components, $X_{0}$ is the spin- 0 scalar, and $\Lambda$ is the EFT energy scale below which the terms are valid. To probe the tensor coupling structure, two decay channels are used [21]. In the $W W \rightarrow e v \mu \nu+0$-jet channel, discriminants such as $m_{l l}, \Delta \phi_{l l}, p_{\mathrm{T}}^{l l}, E_{l l v v}, \Delta p_{\mathrm{T}}$ are used. In the $Z Z \rightarrow 4 l$ channel, the five angles $\theta^{\star}, \Phi_{1}, \Phi, \theta_{1}, \theta_{2}$ and background rejecting $\mathrm{BDT}_{Z Z}$ are used. For fixed CP Higgs test, the $0^{+}$ state is preferred than $0_{h}^{+}$and $0^{-}$in data, as shown in Fig. 12. For the cases of mixtures of SM and BSM terms, the analysis excludes $\kappa$ parameter regions far away from 0 (the SM expected value), as shown in Fig. 12 and listed in Tab. 1.
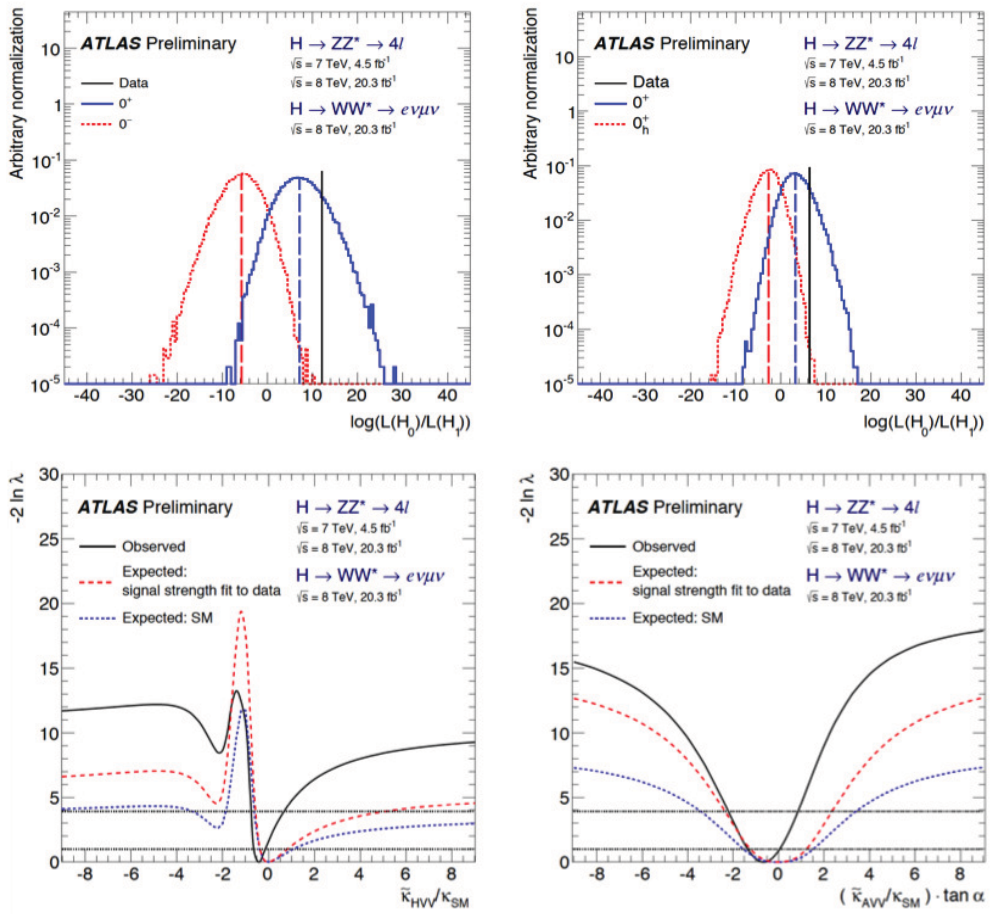

Figure 12. The likelihood ratio distributions to test the fixed Higgs $\mathrm{CP}$ states for $0^{+}$versus $0^{-}$(top left) and $0^{+}$versus $0_{h}^{+}$(top right). For mixed CP states test, the $-2 \ln L$ distributions are shown for SM+BSM-CP-even (bottom left) and SM+BSM-CP-odd (bottom right) cases. Taken from Ref. [21].

Table 1. The combined coupling strength ratio best fit values and 95\% CL excluded regions (see Fig. 12 too), where it is defined that $\tilde{\kappa}_{A V V}=\frac{v}{4 \Lambda} \kappa_{A V V}$ and $\tilde{\kappa}_{H V V}=\frac{v}{4 \Lambda} \kappa_{H V V}$. Taken from Ref. [21].

\begin{tabular}{lcccc}
\hline coupling ratio & \multicolumn{2}{c}{ best fit value } & \multicolumn{2}{c}{$95 \%$ CL exclusion region } \\
& expected & observed & expected & observed \\
\hline$\tilde{\kappa}_{H V V} / \kappa_{\mathrm{SM}}$ & 0.0 & -0.48 & $\leq-0.55$ or $\geq 4.80$ & $\leq-0.73$ or $\geq 0.63$ \\
\hline$\left(\tilde{\kappa}_{A V V} / \kappa_{\mathrm{SM}}\right) \cdot \tan \alpha$ & 0.0 & -0.68 & $\leq-2.33$ or $\geq 2.30$ & $\leq-2.18$ or $\geq 0.83$ \\
\hline
\end{tabular}




\subsection{The Lepton Flavor Violating (LFV) Higgs coupling}

Flavor Changing Neutral Current has been searched for in both the lepton and quark sectors. Due to the experimental constraint from $\mu \rightarrow e \gamma$, it is estimated that $\mathrm{BR}(H \rightarrow e \mu)<\mathrm{O}\left(10^{-8}\right)$. However, the constraint is much less stringent for the LFV decays of $H \rightarrow e \tau, \mu \tau$ (at $\mathrm{O}(10 \%)$ ). The $H \rightarrow \mu \tau$ analysis [22] uses the transverse mass calculated from the $E_{\mathrm{T}}^{\text {miss }}$ and the two leptons to define two signal regions:

1. SR1: $m_{\mathrm{T}}\left(\mu, E_{\mathrm{T}}^{\text {miss }}\right)>40 \mathrm{GeV}, m_{\mathrm{T}}\left(\tau, E_{\mathrm{T}}^{\text {miss }}\right)<30 \mathrm{GeV}$,

2. SR2: $m_{\mathrm{T}}\left(\mu, E_{\mathrm{T}}^{\mathrm{miss}}\right)<40 \mathrm{GeV}, m_{\mathrm{T}}\left(\tau, E_{\mathrm{T}}^{\mathrm{miss}}\right)<60 \mathrm{GeV}$.

The 2-D transverse mass distributions and the MMC mass in the two signal regions are shown in Fig. 13. The extracted signal rate gives $\operatorname{BR}(H \rightarrow \mu \tau)=(0.77 \pm 0.62) \%$ based on the Run 1 data.
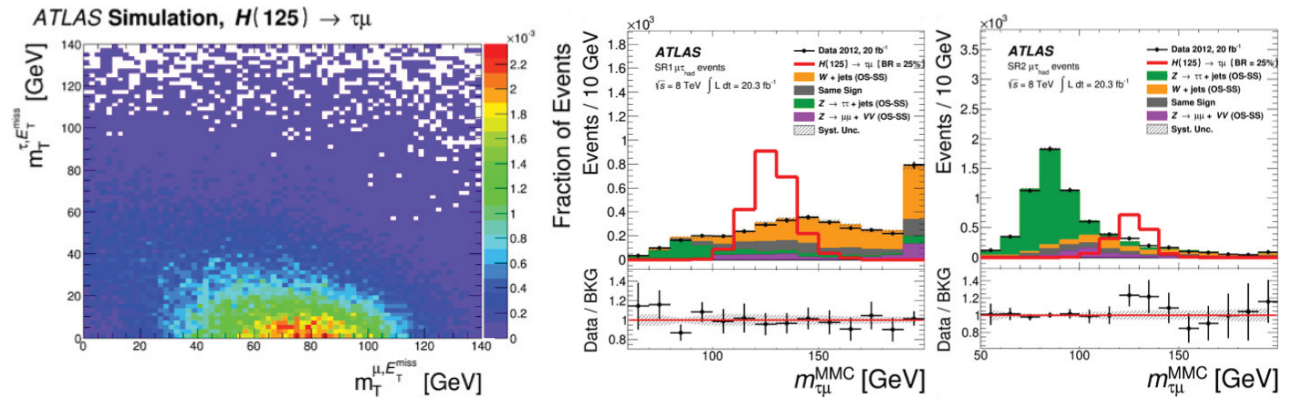

Figure 13. The transverse mass distributions in the signal (left), and the MMC distributions in SR1 (middle) and SR2 (right) signal regions. Taken from Ref. [22].

\section{Higgs prospects}

It is expected that during the lifetime of LHC, there will be about $3000 \mathrm{fb}^{-1}$ of data collected. The current search programs will continue with more data accumulated. With $300 \mathrm{fb}^{-1}$ of Run $2+3$ data, the measurement precision of boson (fermion) coupling will reach a level of about $10 \%$ (20\%), as shown in Fig. 14 [23]. The Higgs mass will be determined more precisely, and the width measurement may become more feasible. New Higgs physics such as the MSSM, rare or new Higgs decays may show up. With more statistics, the differential Higgs distributions, using the EFT theory, can test the Higgs properties to a much deeper level. Fig. 14 also shows the level of precision that can be achieved for Higgs couplings with particles of different masses, with 300 and $3000 \mathrm{fb}^{-1}$ of data.

\section{Conclusion}

After the Higgs boson discovery, the main focus has shifted to its property measurements. This includes the precise mass determination (in the $\gamma \gamma$ and $Z Z$ channels), couplings to different SM particles (bosons and fermions), $\mathrm{CP}$ and tensor coupling structures, total decay width, its BSM partners $\left(H^{ \pm}\right)$ or new decays $(H \rightarrow$ invisible). Many results are improved with advanced analysis methods since the discovery. Combination of different measurements is frequently made to test for any deviations from 

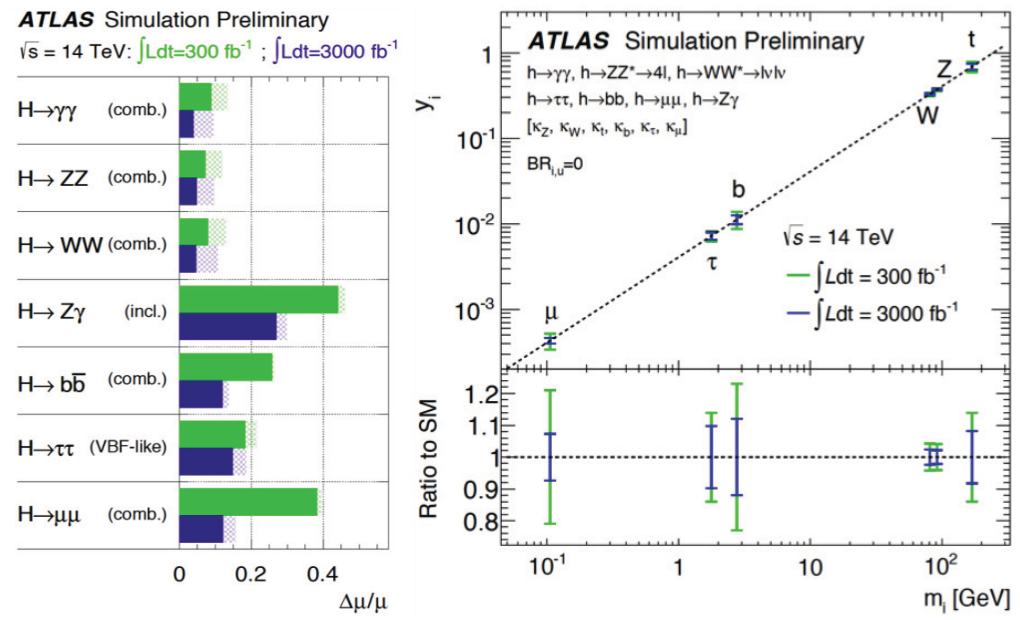

Figure 14. Left: the relative errors on the signal strength measurements in different Higgs decay modes for two luminosity scenarios (the shaded bars represent the systematic errors). Right: the Higgs coupling for particles of different masses for two luminosity scenarios. Taken from Ref. [23].

the SM prediction (so far no significant deviations observed). Our understanding of the new particle has been pushed to an unprecedented level, and will go to a much deeper level with future LHC data. Finally, it should be noted that the Higgs results summarized in this paper are not exhaustive, the analyses techniques will evolve with time, and updated results with more improvements are expected.

\section{References}

[1] ATLAS Collaboration, Phys. Lett. B 716, 1, 2012.

[2] CMS Collaboration, Phys. Lett. B 716, 30, 2012.

[3] ATLAS Collaboration, 2008 JINST 3 S08003.

[4] ATLAS Collaboration, Phys. Rev. D 90, 112015, 2014.

[5] ATLAS Collaboration, Phys. Rev. D 91, 012006, 2015.

[6] ATLAS Collaboration, Phys. Rev. D 90, 052004, 2014.

[7] ATLAS Collaboration, Phys. Rev. D 92, 012006, 2015.

[8] ATLAS Collaboration, JHEP 04, 117, 2015.

[9] A. Elagin et al., Nucl. Instrum. Meth. A 654, 481, 2011.

[10] ATLAS Collaboration, Phys. Lett. B 738, 68, 2014.

[11] ATLAS Collaboration, JHEP 01 069, 2015.

[12] ATLAS Collaboration, Eur. Phys. J. C 75:349, 2015.

[13] ATLAS Collaboration, arXiv:1507.04548.

[14] ATLAS Collaboration, JHEP 03, 088, 2015.

[15] ATLAS Collaboration, Eur. Phys. J. C 75:335, 2015

[16] J.M. Campbell et al., Phys. Rev. D 89, 05301, 2014; JHEP 04, 060, 2014.

[17] ATLAS Collaboration, Phys. Rev. Lett. 112, 201802, 2014.

[18] ATLAS Collaboration, Eur. Phys. J. C 75:337, 2015. 
[19] ATLAS Collaboration, ATLAS-CONF-2015-004, https://cds.cern.ch/record/2002121.

[20] ATLAS Collaboration, arXiv:1509.00672.

[21] ATLAS Collaboration, ATLAS-CONF-2015-008, https://cds.cern.ch/record/2002414.

[22] ATLAS Collaboration, arXiv:1508.03372.

[23] ATLAS Collaboration, ATL-PHYS-PUB-2014-016, https://cds.cern.ch/record/1956710; ATLPHYS-PUB-2014-017, https://cds.cern.ch/record/1956711. 